\title{
Heterodyne near-field scattering: A technique for complex fluids
}

\author{
F. Ferri, ${ }^{1, *}$ D. Magatti, ${ }^{1}$ D. Pescini, ${ }^{1}$ M. A. C. Potenza, ${ }^{2}$ and M. Giglio ${ }^{2}$ \\ ${ }^{1}$ Dipartimento di Fisica e Matematica, Università dell'Insubria and INFM, Via Valleggio 11, Como I-22100, Italy \\ ${ }^{2}$ Dipartimento di Fisica, Università di Milano and INFM, Via Celoria, 16, Milano I-20133, Italy \\ (Received 11 December 2003; revised manuscript received 7 June 2004; published 27 October 2004)
}

\begin{abstract}
We implemented the heterodyne near-field scattering (HNFS) technique [D. Brogioli et al., Appl. Phys. Lett. 81, 4109 (2002)], showing that it is a fairly valid alternative to traditional elastic low-angle light scattering and quite suitable for studying complex fluids such as colloidal systems. With respect to the original work, we adopted a different data reduction scheme, which allowed us to improve significantly the performance of the technique, at levels of sensitivity and accuracy much higher than those achievable with classical low-angle light scattering instrumentation. This method also relaxes the requirements on the optical/mechanical stability of the experimental setup and allows for a real time analysis. The HNFS technique has been tested by using calibrated colloidal particles and its capability of performing accurate particle sizing was ascertained on both monodisperse and bimodal particle distributions. Nonstationary samples, such as aggregating colloidal solutions, were profitably studied, and their kinetics quantitatively characterized.
\end{abstract}

DOI: 10.1103/PhysRevE.70.041405

PACS number(s): 61.43.Hv, 42.25.Fx

\section{INTRODUCTION}

Near-field scattering (NFS) is a family of techniques recently introduced [1-4] in the field of laser light scattering as alternative methods for the measurement of the low-angle scattered intensity distribution. While the traditional lowangle scattering techniques $[5,6]$ collect the scattered light in the far field of the sample and adopt an optical configuration in which there is a one-to-one mapping between the sensor position and the scattering angle, NFS works by collecting the scattered light in the near field of the sample, without any angle-resolved detection scheme. NFS requires a remarkably simple optical setup, in which a large collimated beam is sent onto a square cell containing the sample and the scattered light is detected at a close distance by using a charge-coupled device (CCD) sensor. In this way, each pixel of the sensor is reached by the light scattered at all the angles the system can scatter at, and the angular scattered intensity distribution is retrieved by properly analyzing the statistical properties of the recorded images.

There are three different configurations under which NFS can be realized: (i) a homodyne layout [1,2], in which the transmitted beam is stopped and only the scattered light can reach the sensor; (ii) a heterodyne layout [3] (HNFS) where the transmitted beam is superimposed to the (much weaker) scattered light and the resulting interference pattern is then detected by the sensor; (iii) a Schlieren layout [4] (SNFS), in which the interference between the transmitted beam and the scattered light takes place after a Schlieren-like spatial filter [7] has removed the half plane of the interfering wave vectors. In all these configurations, due to the stochastic interference associated with the scattering waves, the recorded images have a speckled appearance, and, at variance with what happens in the far field, the size of the speckles is independent of the distance between the sample and the sen-

\footnotetext{
*Corresponding author. Email address: fabio.ferri@uninsubria.it
}

sor and from the wavelength of the incident radiation, but it is of the same order as the scatterers' dimension [1,2]. Thus the images contain information on the scatterer structure, which is ultimately related to the scattered intensity distribution. The statistical analysis to be carried out on the recorded images is quite different, depending whether homodyne- or heterodynelike configuration is adopted. The latter (either HNFS or SNFS) have been proved to be much more powerful because they are self-referencing methods, in which the static intense transmitted beam acts as a local oscillator that amplifies the weak fluctuating scattering signal (heterodyne signal), leading to a direct measurement of the scattered field amplitude and allowing intrinsic absolute cross sections to be measured. They also offer the possibility of rigorous stray light subtraction without the necessity of any blank measurement, a feature of fundamental importance when dealing with low-angle scattered light. In the heterodyne-based configurations the data analysis is also quite simple, and the scattered intensity distribution can be recovered as the twodimensional power spectrum of the detected heterodyne signal.

In this article we will focus on the HNFS technique, which exhibits the simplest and most compact optical setup, almost free from any alignment requirements. However, since HNFS is a heterodyne technique in which the level of the signal (scattered light) is typically of the order of a few percent with respect to the level of the local oscillator (transmitted beam), the stability of the latter must be remarkably high. This imposes a rather stringent requirement on the optical/mechanical stability of the setup, which becomes quite demanding when the overall measuring time $T$ is relatively long (minutes). It is this stability that, ultimately, determines the sensitivity and accuracy of the technique.

To overcome this limitation, we developed a procedure for processing the data based on a double-frame differential approach, in which the recovery and the analysis of the heterodyne signal are carried out by comparing two consecutive frames taken at a temporal distance $\Delta t$. In this way, the stability requirements are restricted only to the time distance 


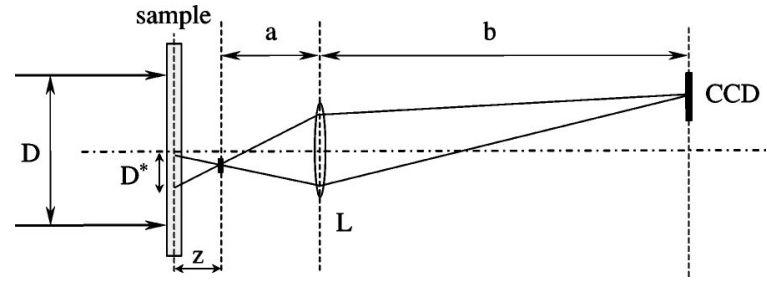

FIG. 1. Schematic diagram of the HNFS setup. The sample is illuminated with a large collimated beam of size $D$ and the transmitted plus scattered light is collected at the sensor plane located at a distance $z$ from the cell. The lens $L$ (a microscope objective) realizes a magnified $(M=b / a)$ image of the sensor plane onto the $\mathrm{CCD}$, which is placed slightly off axis for reducing stray light. $D^{*}$ represents the sample region from which the scattered light is effectively collected.

$\Delta t(\Delta t \ll T)$ and the demands on the optical/mechanical stability of the apparatus are highly relaxed.

We have successfully applied this method to the study of colloidal systems, showing that the technique is quite adequate for performing accurate particle sizing, with a sensitivity much higher than that achievable with traditional lowangle light scattering instrumentation. Moreover, since this method allows for a faster (real time) data analysis, we have also shown that the technique is fairly suitable for studying the kinetics of nonstationary systems, such as the aggregating colloids presented in this work.

\section{THE HETERODYNE NEAR-FIELD SCATTERING TECHNIQUE}

In this section we recall the principles and the practical aspects of the heterodyne near-field scattering technique. We first (Sec. II A) describe the technique as it was originally proposed by some of the authors [3], discussing its main features and great advantages over other classical low-angle scattering instrumentation, but also pointing out some of its limitations, in part associated with the experimental procedure outlined in the original work. Then in Sec. II B we propose a different method of processing the data based on a double-frame differential approach, which relaxes the requirement of stability on the experimental apparatus, allows for a faster (almost real time) data analysis, and improves the overall quality of the data.

\section{A. HNFS, single-frame analysis}

The HNFS techniques works by analyzing the intensity distribution of the light passing through a scattering sample and falling onto a plane located at a close distance $z$ from the cell, as schematically shown in Fig. 1.

In this configuration, the intense static electric field $e_{0}(\mathbf{r})$ associated with the transmitted beam plus stray light acts as a reference beam (local oscillator) and is allowed to interfere with the much weaker time-dependent field $e_{S}(\mathbf{r}, t)$ scattered in the forward direction. The resulting intensity distribution $f(\mathbf{r}, t)$ is therefore composed of a strong static signal due to the main beam intensity on which a weak time-dependent fluctuating ripple, deriving from the interference between the reference and scattered fields (heterodyne term), is superimposed. Any contribution associated with the interference between the scattered waves (homodyne term) is supposed to be negligible $\left(\left|e_{S}\right| \ll\left|e_{0}\right|\right)$.

Thus, by dropping the term $i_{\mathrm{S}}(\mathbf{r}, t)=\left|e_{\mathrm{S}}(\mathbf{r}, t)\right|^{2}$, we can write

$$
f(\mathbf{r}, t)=i_{0}(\mathbf{r})+e_{0}(\mathbf{r}) e_{\mathrm{S}}^{*}(\mathbf{r}, t)+e_{0}^{*}(\mathbf{r}) e_{\mathrm{S}}(\mathbf{r}, t)
$$

where $i_{0}(\mathbf{r})=\left|e_{0}(\mathbf{r})\right|^{2}$. Notice that the static reference term $i_{0}(\mathbf{r})$ depends on $\mathbf{r}$, thus taking into account the spatial dependence of the spurious stray-light contributions always present in a light scattering system operating at low angle. Conversely, the terms $e_{\mathrm{S}}(\mathbf{r}, t)$ and $e_{\mathrm{S}}^{*}(\mathbf{r}, t)$ vary with time and are zero average. Thus, the static contribution $i_{0}(\mathbf{r})$ can be recovered as the time average

$$
i_{0}(\mathbf{r})=\langle f(\mathbf{r}, t)\rangle_{t}
$$

in which the average has to be carried out over a large number of independent sample configurations. Remarkably, Eq. (2) shows that in HNFS a measure of the stray light can be carried out without any effective blank measurement. By subtracting Eq. (2) from Eq. (1), the fluctuating heterodyne signal can be recovered,

$$
\delta f(\mathbf{r}, t) \equiv f(\mathbf{r}, t)-\langle f(\mathbf{r}, t)\rangle_{t}=e_{0}(\mathbf{r}) e_{\mathrm{S}}^{*}(\mathbf{r}, t)+e_{0}^{*}(\mathbf{r}) e_{\mathrm{S}}(\mathbf{r}, t),
$$

and analyzed by computing its Fourier components. For this purpose, let us adopt a notation in which a capital letter represents the spatial Fourier transform $(F)$ of a function indicated with the same small letter $[F\{a(\mathbf{r})\}=A(\mathbf{q})]$, where $\mathbf{q}$ $\equiv\left(q_{x}, q_{y}\right), q_{x}$ and $q_{y}$ being the Fourier vectors associated with the spatial frequencies $f_{x}$ and $f_{y}\left(q_{x}=2 \pi f_{x}, q_{y}=2 \pi f_{y}\right)$. By recalling the Fourier convolution theorem, $\square(a \cdot b)$ $=A * B$, and the relation $\mathrm{F}\left(a^{*}\right)=A *(-\mathbf{q})$, the $\mathrm{F}$ transform of Eq. (3) gives

$$
\delta F(\mathbf{q}, t)=E_{0}(\mathbf{q}) * E_{\mathrm{S}}^{*}(-\mathbf{q}, t)+E_{0}^{*}(-\mathbf{q}) * E_{\mathrm{S}}(\mathbf{q}, t)
$$

where $\delta_{F}=\mathrm{F}\left\{\delta_{f}\right\}$. Equation (4) shows that the q component of the signal observed on the sensor plane is the result of two contributions deriving from the interference between the reference beam and the three-dimensional plane waves scattered with the two vectors $\mathbf{k}_{\mathrm{S}}^{+}=\left(\mathbf{q}, k_{z}\right)$ and $\mathbf{k}_{\mathrm{S}}^{-}=\left(-\mathbf{q}, k_{z}\right)$ and amplitudes $E_{\mathrm{S}}(\mathbf{q}, t)$ and $E_{\mathrm{S}}(-\mathbf{q}, t)$, respectively.

Equation (4) shows also that $\delta F(\mathbf{q}, t)$ carries the information on the scattering field amplitudes only through the convolution with $E_{0}(\mathbf{q})$. However, since we expect that the straylight contributions are definitely smaller than the amplitude of the transmitted beam and constantly increasing at lower and lower $q$, we can reasonably assume that the spectrum $E_{0}(\mathbf{q})$ is much narrower than the spectrum of the scattered light $E_{\mathrm{S}}(\mathbf{q})$ [an assumption rigorously satisfied for the case of an ideal plane-wave local oscillator, for which $E_{0} \sim \delta(\mathbf{q})$ ]. Thus, the convolution appearing in Eq. (4) does not affect significantly the spectra of the scattering fields and we can simplify Eq. (4), obtaining

$$
\delta F(\mathbf{q}, t) \sim E_{\mathrm{S}}^{*}(-\mathbf{q}, t)+E_{\mathrm{S}}(\mathbf{q}, t) .
$$


Equation (4) or (5) holds under the so-called near-field condition $[3,4]$, i.e., when, for any given $\mathbf{q}$, the sensor receives light from a region $D^{*}$ smaller than the illuminated region $D$ (see Fig. 1). Let us denote by $\mathbf{q}_{\max }$ the maximum Fourier vector for which we want Eqs. (4) and (5) to be valid. Thus, the condition $D^{*}<D$ leads to the constraint for the distance $z$ between the sensor and the sample

$$
z<D / 2 \theta_{\max }
$$

in which $\theta_{\max }$ is the scattering angle associated with $\mathbf{q}_{\max }$. Practically, $\theta_{\max }$ or $\mathbf{q}_{\max }$ is determined either by the (imaged) pixel size of the CCD sensor, or by the numerical aperture (NA) associated with the objective used for collecting the scattered light, whichever is smaller. In the case of a typical HNFS, the bottleneck is usually represented by the objective NA. For example, for the case of the $20 \times$ objective used in this work (see next section), the numerical aperture was $\mathrm{NA}=0.50$ corresponding to $\theta_{\max }=30^{\circ}$. The corresponding maximum allowed distance was therefore $z<10 \mathrm{~mm}$.

The power spectrum of the heterodyne signal is obtained by squaring Eq. (5), giving

$$
\begin{aligned}
|\delta F(\mathbf{q}, t)|^{2} \sim & \left|E_{\mathrm{S}}(\mathbf{q}, t)\right|^{2}+\left|E_{\mathrm{S}}(-\mathbf{q}, t)\right|^{2}+E_{\mathrm{S}}(-\mathbf{q}, t) E_{\mathrm{S}}(\mathbf{q}, t) \\
& +E_{\mathrm{S}}^{*}(-\mathbf{q}, t) E_{\mathrm{S}}^{*}(\mathbf{q}, t) .
\end{aligned}
$$

The first two terms of Eq. (7) are identical to each other and can be directly related to the scattered intensity distribution to be recovered (see below). The last two terms are the socalled shadowgraph terms [8], and are responsible for deep oscillations appearing in the low- $q$ region of the spectrum. These oscillations arise from the fact that the waves scattered with $\mathbf{q}$ and $-\mathbf{q}$ may be partially correlated, with the phase difference depending on both $q=|\mathbf{q}|$ and $z$. It is beyond the purpose of this paper to discuss this effect in somewhat more detail and we refer the reader to Ref. [3,9]. It is here sufficient to mention that, when $z$ and/or $q$ are large enough, the phase difference of the $\mathbf{q}$ and $-\mathbf{q}$ waves becomes random, and the two correlation terms appearing in Eq. (7) vanish when averaged over time. In the framework of this article we will always neglect such terms.

For stationary isotropic samples we can average Eq. (7) and obtain the mean spectrum

$$
S(q)=\left\langle|\delta F(\mathbf{q}, t)|^{2}\right\rangle_{t, q}
$$

in which the $t$ average has to be carried out over a time long enough to accumulate a large number of independent sample configurations, while the $q$ average is performed over all the vectors $\mathbf{q}$ such that $\sqrt{q_{x}^{2}+q_{y}^{2}}=q$.

The final step in the HNFS procedure is to recall the relation between the spectrum $S(q)$ and the scattered intensity distribution $I_{\mathrm{S}}(Q)$, which is customarily reported as a function of the modulus of the transferred wave vector $\mathbf{Q}$, defined as the difference between the scattered wave vector $\mathbf{k}_{\mathrm{s}}$ and the incident wave vector $\mathbf{k}_{0}$, i.e., $\mathbf{Q}=\mathbf{k}_{\mathrm{s}}-\mathbf{k}_{0}$. Since the scattering is elastic $\left(\left|\mathbf{k}_{\mathrm{s}}\right|=\left|\mathbf{k}_{0}\right|=k\right)$, we have $Q=2 k \sin (\theta / 2), \theta$ being the scattering angle. The vector $\mathbf{Q}$ is simply related to $\mathbf{q}$ and, with the help of Fig. 2, it is immediately easy to work out that

$$
Q=\sqrt{2} k\left[1-\sqrt{1-(q / k)^{2}}\right]^{1 / 2},
$$

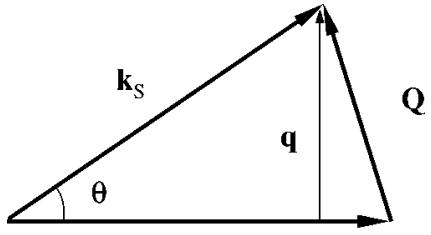

$\mathbf{k}_{0}$

FIG. 2. Vectors and angles involved in the HNFS technique. $\mathbf{k}_{0}$, incident wave vector; $\mathbf{k}_{\mathrm{S}}$, scattered wave vector; $\mathbf{Q}$, transferred wave vector; q, Fourier vector; $\theta$, scattering angle.

$$
q=Q \sqrt{1-(Q / 2 k)^{2}},
$$

which reduces to $Q \sim q$ in the limit $\theta \rightarrow 0$.

By using Eq. (8) it is now possible to express the scattered intensity distribution as

$$
I_{\mathrm{S}}(Q) \sim S[q(Q)]
$$

the same recovered with a traditional low-angle scattering apparatus.

As already mentioned in the Introduction, HNFS is a powerful technique which exhibits many remarkable features like a rigorous subtraction of the stray light, almost no requirement on the optical alignment, and simplicity and compactness of the setup. However, there are also some nonsecondary limitations which may hamper the actual applicability of the technique. The most important one is related to the requirements on the optical/mechanical stability of the setup. Since typically the rms level of the signal fluctuations is of the order of $1 \%$ with respect to the static background, and the overall measuring time can be as long as several minutes, we are imposing a rather stringent requirement on the stability of the reference term $i_{0}(\mathbf{r})$. A slow drift of the laser power, or a slight change in the alignment induced by mechanical/thermal relaxations, or any other reason causing a change of the optical background during the measuring time, could produce variations of $i_{0}(\mathbf{r})$ stronger than or comparable with the weak intensity fluctuations we want to measure. Thus, Eq. (2) does not hold anymore, and the technique may become highly inaccurate. An example of that will be given in Fig. 4, which will be discussed at the end of Sec. II B. Another limitation is the off-line analysis associated with the procedure. First, many independent frames must be acquired and stored, and only afterward can the analysis be carried out.

\section{B. HNFS, double-frame analysis}

The limitations associated with the single-frame analysis discussed above can be removed by adopting a differential double-frame analysis. Suppose that we acquire a set of different frames corresponding to many independent sample configurations, and let $\Delta t$ be the temporal distance between each frame. Thus, indicating with $f_{1}$ and $f_{2}$ the frames taken at time $t$ and $t+\Delta t$, respectively, under the same assumptions of Eq. (1), we can write

$$
f_{1}(\mathbf{r}, t)=i_{0}(\mathbf{r})+e_{0}(\mathbf{r}) e_{1}^{*}(\mathbf{r}, t)+e_{0}^{*}(\mathbf{r}) e_{1}(\mathbf{r}, t)
$$




$$
f_{2}(\mathbf{r}, t, \Delta t)=i_{0}(\mathbf{r})+e_{0}(\mathbf{r}) e_{2}^{*}(\mathbf{r}, t+\Delta t)+e_{0}^{*}(\mathbf{r}) e_{2}(\mathbf{r}, t+\Delta t)
$$

and their difference

$$
\begin{aligned}
\delta f(\mathbf{r}, t, \Delta t)= & f_{2}(\mathbf{r}, t+\Delta t)-f_{1}(\mathbf{r}, t) \\
= & e_{0}(\mathbf{r}) e_{2}^{*}(\mathbf{r}, t+\Delta t)+e_{0}^{*}(\mathbf{r}) e_{2}(\mathbf{r}, t+\Delta t) \\
& -e_{0}(\mathbf{r}) e_{1}^{*}(\mathbf{r}, t)-e_{0}^{*}(\mathbf{r}) e_{1}(\mathbf{r}, t)
\end{aligned}
$$

is clearly independent of $i_{0}(\mathbf{r})$. By following the same analysis outlined in Sec. II A, we obtain

$$
\begin{aligned}
\delta F(\mathbf{q}, t, \Delta t) \sim & {\left[E_{2}^{*}(-\mathbf{q}, t+\Delta t)+E_{2}(\mathbf{q}, t+\Delta t)\right] } \\
& -\left[E_{1}^{*}(-\mathbf{q}, t)+E_{1}(\mathbf{q}, t)\right],
\end{aligned}
$$

and by squaring Eq. (13) we have

$$
|\delta F(\mathbf{q}, t, \Delta t)|^{2}=\left|\alpha_{1}\right|^{2}+\left|\alpha_{2}\right|^{2}+\alpha_{1} \alpha_{2}^{*}+\alpha_{1}^{*} \alpha_{2},
$$

where $\quad \alpha_{1}=E_{1}^{*}(-\mathbf{q}, t)+E_{1}(\mathbf{q}, t) \quad$ and $\quad \alpha_{2}=E_{2}^{*}(-\mathbf{q}, t+\Delta t)$ $+E_{2}(\mathbf{q}, t+\Delta t)$. Clearly, for a stationary sample, the terms $\left|\alpha_{1}\right|^{2}$ and $\left|\alpha_{2}\right|^{2}$ are identical and equal to the expression reported in Eq. (7), and are related in the same way to the scattered intensity distribution $I_{\mathrm{S}}(Q)$ [see Eq. (10)]. Conversely, the terms $\alpha_{1} \alpha_{2}^{*}$ and $\alpha_{1}^{*} \alpha_{2}$ describe the spatial correlations between the two frames taken at a temporal distance $\Delta t$, and, for uncorrelated frames, they vanish. The latter point is rather critical and consequently the frame distance $\Delta t$ must be tuned quite finely: on one hand it has to be large enough so as to ensure decorrelation between the fluctuating components of the two frames; on the other hand it cannot be too large because, otherwise, we fall again into the framework of Sec. II A, with its limitations arising from the long measuring time.

It is worth mentioning at this time an important point about future developments of the HNFS technique. When $\Delta t$ is smaller than the correlation time between the two frames, the terms $\alpha_{1} \alpha_{2}^{*}$ and $\alpha_{1}^{*} \alpha_{2}$ do carry information on the dynamics of the system. Thus, by properly analyzing such terms as a function of the (lag) time $\Delta t$, one should be able to extract the time-correlation function of the scattered intensity, simultaneously, at all the $\mathbf{q}$ vectors. Thus simultaneous measurements of dynamic and static light scattering would became feasible, greatly enlarging the potentialities of the HNFS technique.

In conclusion, whenever the time $\Delta t$ is much shorter than the overall measuring time $T=N \Delta t$, with $N \gg 1$ being the number of independent frames, the double-frame procedure is expected to work better that the single-frame method. A quantitative comparison between the two procedures will be reported in Sec. IV A.

\section{OPTICAL SETUP}

The measurements were performed by using the heterodyne optical setup sketched in Fig. 1. A cw He-Ne laser (wavelength in the vacuum $632.8 \mathrm{~nm}$ ) was spatially filtered, collimated to a diameter $D \sim 10 \mathrm{~mm}$ (at $1 / e^{2}$ of the intensity) and sent onto the sample, which was contained in a rectan- gular quartz cell, $2 \mathrm{~mm}$ optical path, with walls $1 \mathrm{~mm}$ thick. The collecting lens was a $20 \times$ Spindler \& Hoyer microscope objective with a numerical aperture NA $=0.50$, to which corresponds a maximum transferred wave vector $Q_{\max }$ $\sim 4.5 \mu \mathrm{m}^{-1}$. The objective was positioned at a distance from the exit face of the cell of $1 \mathrm{~mm}$, implying that the observation plane was at a distance from the center of the cell of about $z=1.4 \mathrm{~mm}$, which is compliant with the near-field condition Eq. (6).

The images were acquired by using a 12-bit digital CCD camera (Vooskuhler CCD-1300, $1280 \times 1024$ pixel, pixel size $6.7 \mu \mathrm{m}$ ) interfaced to the PC by means of a digital frame grabber (National Instrument, model PCI-1422). The camera was positioned slightly off axis to reduce stray light. Only a square portion $1024 \times 1024$ of the image was used in the Fourier analysis (fast Fourier transform). The objective magnification $20 \times$ ensures that the sizes of the speckles, being of the order of the particle size, are larger than the dimensions of the CCD pixels. The magnification determines also the effective side of the sensor $L=6.7 \mu \mathrm{m} \times(1024 / 20)$ $\sim 343 \mu \mathrm{m}$ and, correspondingly, the minimum detectable wave vector $Q_{\min }=2 \pi / L \sim 1.83 \times 10^{-2} \mu \mathrm{m}^{-1}$.

\section{EXPERIMENTAL RESULTS}

The performance of the HNFS technique was evaluated by carrying out several tests on diluted solutions of calibrated, almost monodisperse $(\sigma /\langle d\rangle \sim 1 \%)$, polystyrene spheres (Duke Scientific Co.). We started by comparing the single- and double-frame analysis (Sec. IV A); then we investigated how the sensitivity of the HNFS technique compares with the one allowed by a standard low-angle light scattering instrument (Sec. IV B); the next test was aimed at ascertaining the accuracy of the HNFS technique and its capability of performing reliable particle sizing (Sec. IV C); finally, we investigated the suitability of the technique for studying nonstationary samples, such as aggregating colloids (Sec. IV D).

\section{A. Comparison between single- and double-frame analysis}

A qualitative but striking difference between the singleand double-frame analyses is illustrated in Fig. 3, which refers to measurements taken on a sample of $5 \mu \mathrm{m}$ diameter particles, after having accumulated $N=60$ frames at $\Delta t=5 \mathrm{~s}$. In order to emphasize the differences, we intentionally picked up a set of data that, once analyzed with the singleframe procedure, turned out to be fairly noisy. This effect is shown in the first row of Fig. 3 (frames A,B,C) which reports the average frame $\langle f\rangle_{t}(\mathrm{~A})$, the first frame $f_{1}(\mathrm{~B})$, and their difference $\langle f\rangle_{t}-f_{1}(\mathrm{C})$. Both the average and the single frame exhibit evident spurious patterns and fringes due to stray light, which remain (at a much lower rms level) in their difference also. Conversely, in the double-frame procedure (second row, D,E,F), the stray-light subtraction is very accurate and the frame difference $f_{2}-f_{1}(\mathrm{~F})$ appears to be a fairly regular speckle field, with no reminiscence of the original stray pattern and fringes. 

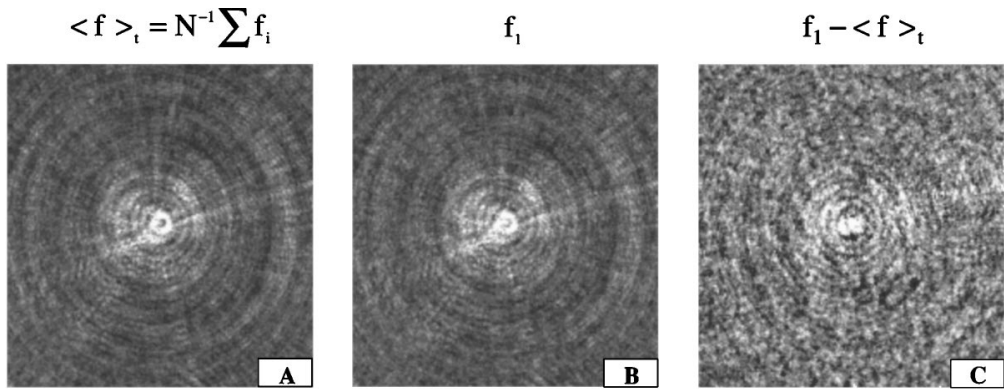

$\mathbf{f}_{1}$

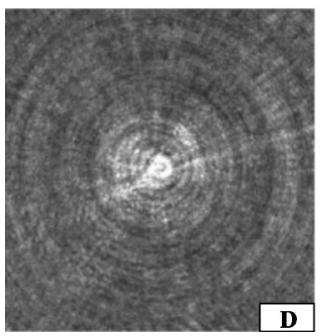

$\mathbf{f}_{2}$

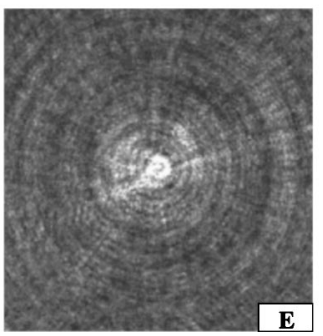

$$
\mathrm{f}_{2}-\mathrm{f}_{1}
$$

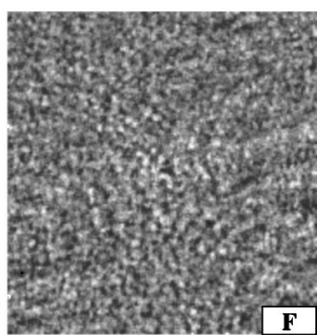

FIG. 3. Comparison between the single-frame (first row, A,B,C) and double-frame (second row, D,E,F) data analyses. The sample was a $5 \mu \mathrm{m}$ (diameter) particle solution and the data were accumulated for $N=60$ frames at $\Delta t=5 \mathrm{~s}$. The frame difference $f_{2}-f_{1}(\mathrm{~F})$ of the double-frame analysis appears to be much more effective than the corresponding difference $f_{1}-\langle f\rangle_{t}(\mathrm{C})$ associated with single-frame analysis.
A much more quantitative comparison can be carried out by plotting together the two recovered scattered distributions, as shown in Fig. 4 in which the $I_{\mathrm{S}}(Q)$ associated with single and double-frame procedures are represented as squares and circles, respectively. Notice that the two analyses were carried out on the very same set of data. The distribution derived from the double-frame procedure is much smoother than the other one, and matches fairly accurately the theoretical curve computed using the Mie theory.

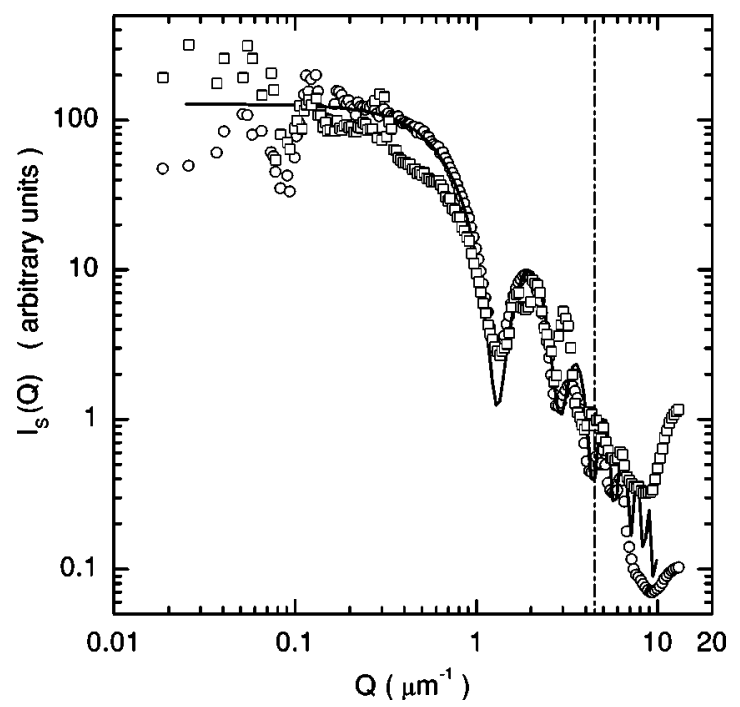

FIG. 4. Comparison between the scattered intensity distributions recovered by using the single-frame (squares) and the double-frame (circles) analyses. Sample and raw data were the same as in Fig. 3 ( $5 \mu \mathrm{m}$ particle, $N=60$ frames at $\Delta t=5 \mathrm{~s}$ ). The two analyses have been carried out on the same set of data. The distribution obtained with the double-frame analysis is much smoother and matched accurately the expected Mie curve (solid curve). The vertical dashdotted line indicates the maximum wave vector allowed by the numerical aperture of the objective $(\mathrm{NA}=0.5)$.

\section{B. Sensitivity of the HNFS technique}

As explained in Sec. II A, the HNFS technique is based on the interference between the static intense transmitted beam $e_{0}$ and the weak fluctuating scattering field $e_{\mathrm{S}}$. Thus $\left|e_{S}\right| \ll\left|e_{0}\right|$, and the interference between the two fields gives rise to a heterodyne signal which is much stronger than the corresponding homodyne signal. Notice that the latter is the quantity that is measured in a standard light scattering instrument.

We can quantify the relative strength between the heterodyne and homodyne signals in the following way. Suppose that the sample is a solution of $N$ identical particles contained in a thin cell and let $z$ be the distance between the sensor plane and the cell (see Fig. 1). Thus, the scattered field is the sum of $N$ contributions

$$
e_{\mathrm{S}}(\mathbf{r}, t)=\frac{1}{k z} \sum_{i=1}^{N} a_{i}(\mathbf{r}, t) e^{-j \phi_{i}(\mathbf{r}, t)} e_{0},
$$

in which $a_{i}$ and $\phi_{i}$ are the amplitude and the phase of the scattered field associated with the $i$ th particle. Note that both $a_{i}$ and $\phi_{i}$ are time dependent because of particle motion. By using Eq. (15), we can rewrite Eq. (1) and, without dropping the homodyne term, obtain

$$
\begin{aligned}
f(\mathbf{r}, t)= & i_{0}(\mathbf{r})\left[1+\frac{2}{k z} \operatorname{Re}\left(\sum_{i=1}^{N} a_{i}(\mathbf{r}, t) e^{-j \phi_{i}(\mathbf{r}, t)}\right)\right. \\
& \left.+\frac{1}{k^{2} z^{2}}\left|\sum_{i=1}^{N} a_{i}(\mathbf{r}, t) e^{-j \phi_{i}(\mathbf{r}, t)}\right|^{2}\right]
\end{aligned}
$$

where the second and third terms in the square brackets are the heterodyne and homodyne signals, respectively. If the particles are randomly distributed inside the cell, the phases $\phi_{i}$ vary stochastically, and, in the limit of $N \gg 1$, the sum behaves as a two-dimensional random walk. Thus 


$$
\sum_{i=1}^{N} a_{i}(\mathbf{r}, t) e^{-j \phi_{i}(\mathbf{r}, t)} \sim \sqrt{N}\langle a\rangle e^{-j \psi(\mathbf{r}, t)}
$$

where $\psi(\mathbf{r}, t)$ is a random phase varying between 0 and $2 \pi$ and $\langle a\rangle$ is the average amplitude of the scattered field given by

$$
\langle a\rangle=\left\langle a_{i}(\mathbf{r}, t)\right\rangle_{N}=\theta_{\max }^{-1} \int_{0}^{\theta_{\max }} a(\theta) d \theta,
$$

where $\theta_{\max }$ is the maximum scattering angle associated with the optics. Note that, because of ergodicity, $\langle a\rangle$ is independent of both sensor position and time.

By using Eq. (1) and working out the last term of Eq. (16), the heterodyne and homodyne signals become

$$
\begin{gathered}
f(\mathbf{r}, t)_{\text {heter }}=\frac{2 \sqrt{N}\langle a\rangle}{k z} \cos [\psi(\mathbf{r}, t)], \\
f(\mathbf{r}, t)_{\text {homo }}=\frac{N\left\langle a^{2}\right\rangle}{k^{2} z^{2}}+\frac{2\left\langle a^{2}\right\rangle}{k^{2} z^{2}} \sum_{i>j}^{N} \cos \left[\Delta \phi_{i j}(\mathbf{r}, t)\right],
\end{gathered}
$$

in which $\Delta \phi_{i j}(\mathbf{r}, t)=\phi_{i}(\mathbf{r}, t)-\phi_{j}(\mathbf{r}, t)$. As expected both terms fluctuate stochastically in time, but while the heterodyne signal is zero average, for the homodyne one the average value is $N\left\langle a^{2}\right\rangle / k^{2} z^{2}$. Equation (19) shows the remarkable difference between the strengths of these two terms: the rms amplitude of the heterodyne signal scales as the square root of the average homodyne signal. Thus, since both terms are much smaller than unity, and is the first one is much stronger than the second one, characterized by a much more compressed dynamics. This is the key point that makes the HNFS technique (heterodyne signal) much more sensitive than the traditional light scattering technique (homodyne signal).

We quantitatively compared the sensitivity of the HNFS technique with the one associated with a classical low-angle light scattering (LALS) apparatus by measuring the intensity distribution $I_{\mathrm{S}}(Q)$ scattered by a solution of $3 \mu \mathrm{m}$ latex particles at increasingly lower concentrations. For the HNFS technique we adopted the same parameters used in Sec. IV A and processed the data following the double-frame analysis. The LALS measurements were carried out by using a state of the art instrument [5] and the $I_{S}(Q)$ distributions were recovered by following the usual procedure in which a blank measurement, taken with the cell filled only with solvent, is subtracted from a sample measurement taken with the cell containing the scattering solution.

The sample concentrations were varied between $\phi$ $\sim 2 \times 10^{-5}$ and $6 \times 10^{-8}$ volume fractions, corresponding to remarkably low beam attenuations (over a $2 \mathrm{~mm}$ optical path), of the order of $\sim 4 \times 10^{-2}-10^{-4}$. For each concentration, the comparison was carried out on the very same sample, i.e., by using the same scattering cell and taking the LALS and HNFS measurements one immediately after the other. The results are shown in Fig. 5, in which the solid symbols refer to the LALS data, while the open symbols describe the HNFS data. Next to each curve, the expected values for the beam attenuations are also indicated. The fig-

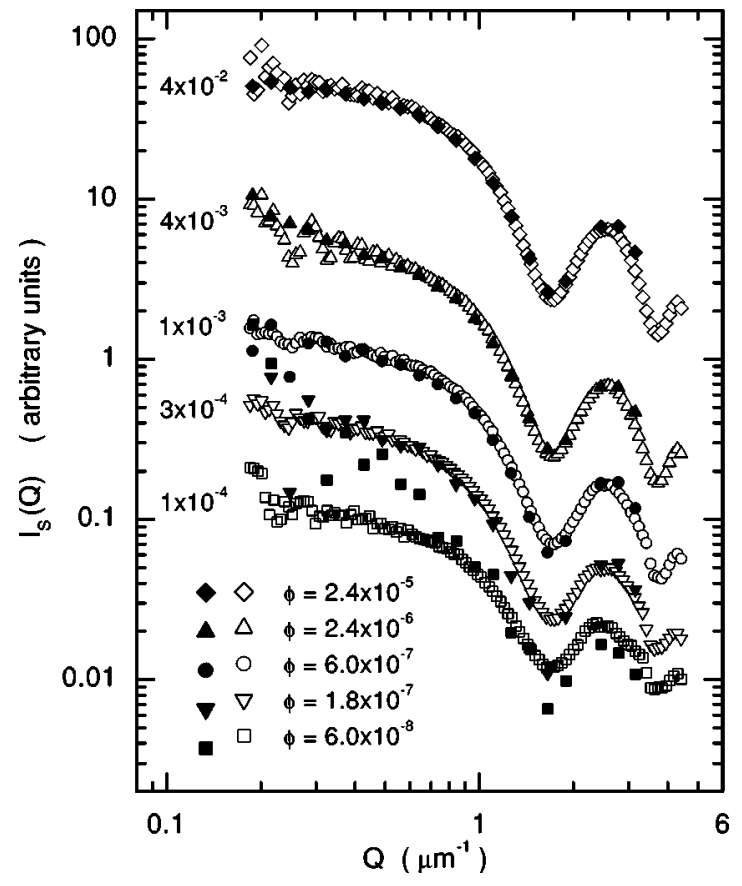

FIG. 5. Comparison between the scattered intensity distributions recovered by HNFS technique (open symbols) and by a state of the art low-angle light scattering apparatus (solid symbols). The sample was a solution of $3 \mu \mathrm{m}$ diameter latex particles at different volume fractions $\phi$. The number by each data set indicates the expected beam attenuation.

ure shows clearly that the two techniques are comparable only at higher concentrations, but as the concentration is reduced, the LALS data become progressively much noisier than the corresponding HNFS data. In particular, while for the LALS technique the minimum concentration at which $I_{\mathrm{S}}(Q)$ can be reliably measured is the one corresponding to a beam attenuation of $\sim 10^{-3}$, for HNFS, the sensitivity is much higher and concentrations of about a factor of 10 lower can be accurately recovered.

It should also be pointed out that the LALS data reported in Fig. 5 have been taken, in some sense, under ideal conditions, in which the stray-light subtraction was extremely effective: the sample measurements were taken immediately after the blank measurements, without moving the scattering cell. The sample was loaded by adding a concentrated solution of colloids to the water already present in the cell (and used for the blank measurement). When we adopted other loading procedures which required moving and repositioning the cell into the holder (which might be a typical situation), the stray-light subtraction was less effective and the LALS data became much noisier (data not shown in Fig. 5). As a matter of fact, only the $I_{\mathrm{S}}(Q)$ at the higher concentrations $\left(\phi \geqslant 10^{-6}\right)$ could be reliably recovered and, in some cases, only for the larger angles.

Conversely, the limitation associated with the stray-light subtraction, which is unavoidable in any LALS instrument, is completely removed in HNFS, which indeed allows for a rigorous subtraction of the stray light, without the necessity of any blank measurement. 

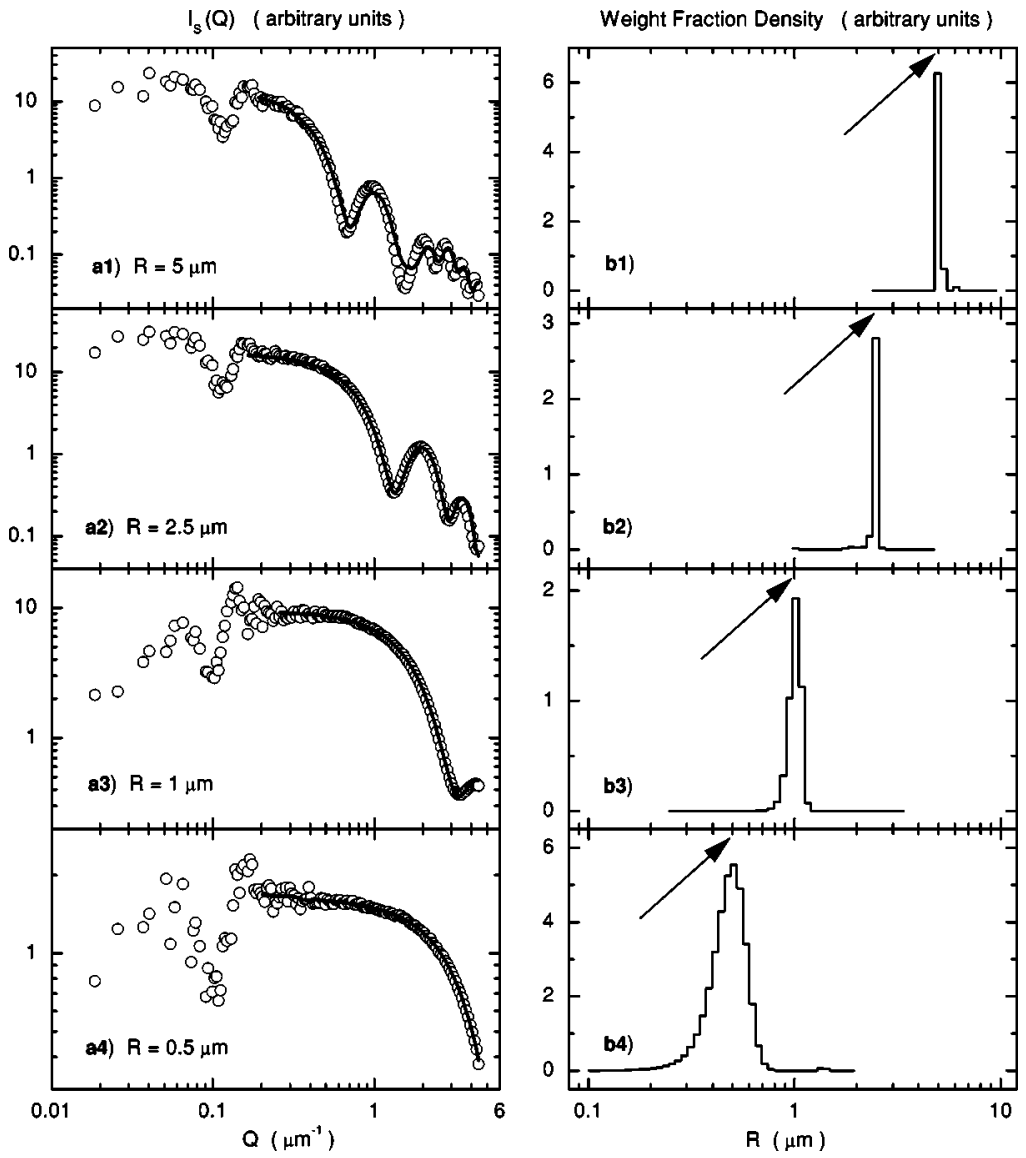

FIG. 6. Scattered intensities of stable monodisperse colloidal particles with diameters in the range 1-10 $\mu \mathrm{m}$ [left column (a)]. Particle size distributions expressed as weight fraction densities [right column (b)] obtained by inverting the corresponding data of column (a). The arrows indicate the values for the expected (certified) radii. In the frames of column (a), the open symbols represent the experimental data, while the solid curves are the intensities reconstructed on the basis of the corresponding recovered distributions. Data with $Q<0.2 \mu \mathrm{m}^{-1}$ are affected by shadowgraph oscillations and were not considered in the inversion.

\section{Particle sizing of stable colloids}

The accuracy of the HNFS technique in recovering the correct scattered intensity distribution $I_{\mathrm{S}}(Q)$ was ascertained by performing particle sizing on stable colloidal solutions prepared by using almost monodisperse $(\sigma /\langle d\rangle \sim 1 \%)$ polystyrene spheres with certified diameters ranging between 1 and $10 \mu \mathrm{m}$ (Duke Scientific Co.). The solutions were diluted to volume fractions ranging between $\sim 10^{-5}$ and $10^{-6}$, so to have beam attenuations of the order of $\sim 1 \%$. For this set of measurements, the images were grabbed every $\Delta t=5 \mathrm{~s}$ and processed following the double-frame analysis. A total number of $N=60$ images were taken, corresponding to an overall measuring time $T=300 \mathrm{~s}$.

For each sample, the measured scattered intensities were processed by using an inversion algorithm that allows the recovery of the sample radii distribution according to the integral equation

$$
I_{\mathrm{S}}(Q)=\int I_{\mathrm{Mie}}(Q, R)[M(R)]^{-1} W(R) d R,
$$

where $I_{\text {Mie }}(Q, R)$ is the intensity scattered by a particle of radius $R$ at the wave vector $Q$, computed according to the Mie theory, $M(R)=(4 / 3) \rho \pi R^{3}$ is the particle mass $(\rho$ being the density), and $W(r) d r$ is the mass of particles with radii between $r$ and $r+d r$. In Eq. (20) $I_{\mathrm{S}}(Q)$ represents the known term (provided by the experiment), $I_{\text {Mie }}(Q, R)[M(R)]^{-1}$ the kernel, and $W(R)$ the unknown distribution to be retrieved. A detailed description of the algorithm and its application to the inversion of low-angle scattering data can be found in Refs. [10,11].

The experimental results are shown in Fig. 6, in which we have reported, on the left column, as a function of the wave vector $Q$ the measured scattered intensities (open symbols) together with the intensities reconstructed on the basis of the recovered distributions (solid curves). The particle distributions are reported on the right column as weight fraction density distributions. The data with $Q<0.2 \mu \mathrm{m}^{-1}$, where shadowgraph oscillations are fairly evident, were not considered for the inversions. All the retrieved distributions are peaked in correspondence with the expected nominal radii (shown by the arrows in the figure, right column) and, with the exception of the $R=0.5 \mu \mathrm{m}$ radius where some limitations of the inversion algorithm show up, appear to be quite narrow, as expected for almost monodisperse distributions. The accuracies of the recovered average radii were of the order of a few percent. Similarly, the reconstructed intensities $I_{\mathrm{S}}(Q)$ matched the experimental data, quite well with deviations of a few percent (rms).

As a second test we considered two bimodal distributions: the first one was a mix of $R_{1}=5.0 \mu \mathrm{m}$ and $R_{2}=2.5 \mu \mathrm{m}$ particles, with corresponding weight fractions $W_{1}=0.62$ and $W_{2}=0.38$; the second distribution had $R_{1}=2.5 \mu \mathrm{m}$ and $R_{2}$ $=1.5 \mu \mathrm{m}$, and $W_{1}=0.80$ and $W_{2}=0.20$. The results are reported in Fig. 7, following the same scheme adopted for Fig. 6. As evident, the reconstructed intensities are recovered quite accurately and the retrieved distributions are fairly narrow and peaked at the right positions, as shown by the arrows. 

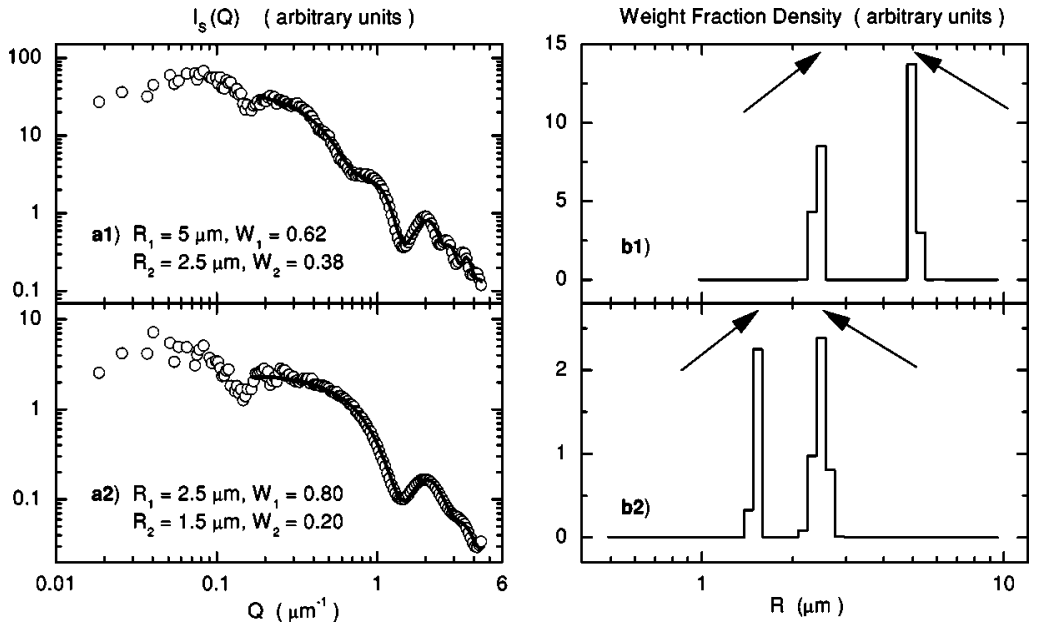

FIG. 7. Scattered intensities of stable bimodal colloidal distributions with particles having diameters different by a factor of about 2 [left column (a)] and corresponding recovered particle size distributions [right column (b)]. Particle radii and corresponding weight fractions are shown on column (a). Same considerations as those reported in Fig. 6 apply.

\section{Kinetics of aggregating colloids}

The suitability of the technique for characterizing nonstationary systems such as polymerizing or gelling solutions, nucleation processes, or systems undergoing phase transitions, was investigated by studying the kinetics of a typical colloidal aggregation. The colloids were latex spheres $70 \mathrm{~nm}$ in diameter (Duke Scientific Co.) at a number concentration $c_{0}=5.6 \times 10^{10} \mathrm{~cm}^{-3}$ (volume fraction $10^{-5}$ ), and the aggregation was induced by adding the divalent salt $\left[\mathrm{MgCl}_{2}\right]$ $=15 \mathrm{mM}$. Under these conditions, the aggregation developed rather slowly (several hours) following the modality of a reaction limited aggregation (RLA), for which the mass fractal dimension is expected to be $D_{\mathrm{m}}=2.1$ [12]. The scattered intensities taken at different times after the addition of salt are shown in Fig. 8 (open symbols), in which for clarity we have reported only the $Q$ range not affected by the shadowgraph oscillations. Each curve was obtained by processing an adequate number of frames as outlined in Sec. II B. In order to avoid correlations between consecutive frames, the temporal distance between them was increased as aggregation was going on, passing from $\Delta t=5 \mathrm{~s}$ for the early times to $\Delta t=200 \mathrm{~s}$ for the final times. The figure shows the typical behavior expected for the evolution of the scattered intensities in a colloidal aggregation experiment: a strong ( $\sim 3$ decades) increase of the zero- $Q$ scattered intensity, accompanied by a remarkable change in the shape of $I_{\mathrm{S}}(Q)$, with the curve roll-off moving toward small $Q$ and the large $Q$ data lying on the same asymptote. The latter is the signature of the aggregate fractal morphology, and represents a measure of their mass fractal dimension $D_{\mathrm{m}}$, because asymptotically $(q \rightarrow \infty) I_{\mathrm{S}} \sim Q_{\mathrm{S}}{ }^{-D_{\mathrm{m}}}$. The data of Fig. 8 were fitted to the so called Fisher-Burford function [13]

$$
I_{\mathrm{S}}(Q)=\frac{I_{\mathrm{S}}(Q=0)}{\left[1+\left(2 / 3 D_{\mathrm{m}}\right) R_{\mathrm{G}}^{2} Q^{2}\right]^{D_{\mathrm{m}} / 2}}
$$

in which the fitting parameters were the zero- $Q$ intensity $I_{\mathrm{S}}(Q=0)$, the fractal dimension $D_{\mathrm{m}}$, and the cluster gyration radius $R_{\mathrm{G}}$. The fittings, reported in Fig. 8 as solid curves, are quite satisfactory and allowed us to estimate $D_{\mathrm{m}}=2.1 \pm 0.1$, as expected for RLA.
Since the parameter $I_{\mathrm{S}}(Q=0)$ is proportional to the (weight average) mass cluster $M_{\mathrm{w}}$, we can also probe the relation between $R_{\mathrm{G}}$ and $M_{\mathrm{w}}$ for a fractal cluster, i.e.,

$$
M_{\mathrm{w}} \sim\left(R_{\mathrm{G}}\right)^{D_{\mathrm{m}}}
$$

The behavior predicted by Eq. (22) is confirmed by the data shown in Fig. 9, in which the log-log plot of $I_{\mathrm{S}}(Q=0)$ vs $R_{\mathrm{G}}$ shows a nice (asymptotic) scaling, characterized by the expected exponent $D_{\mathrm{m}}=2.1$.

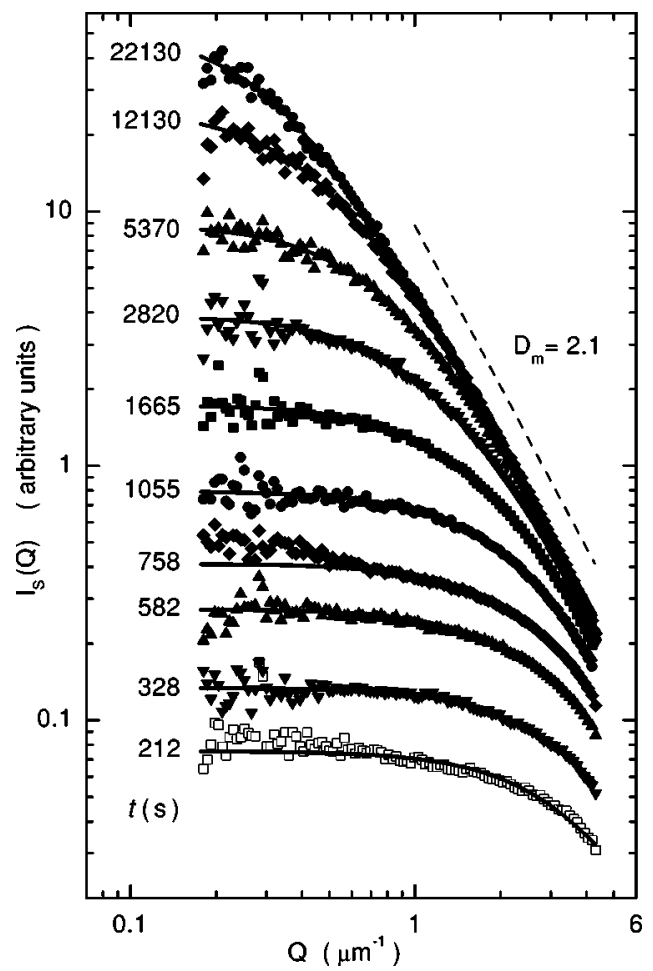

FIG. 8. Scattered intensity distributions of aggregating colloids (70 $\mathrm{nm}$ in diameter) at different times after destabilization with salt. The solid curves represent the fitting of the experimental data to Eq. (21). The dashed straight line shows the slope corresponding to a mass fractal dimension $D_{\mathrm{m}}=2.1$. For the sake of clarity, data affected by shadowgraph oscillations $\left(Q<0.2 \mu \mathrm{m}^{-1}\right)$ have not been reported. 


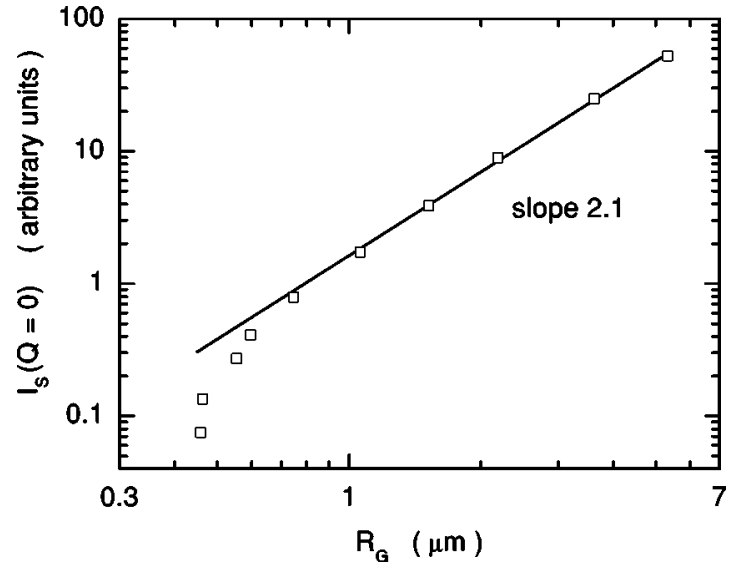

FIG. 9. Scaling between the zero- $Q$ scattered intensity and the gyration radius of the aggregating colloids of Fig. 8. The slope of 2.1 coincides with the mass fractal dimension $D_{\mathrm{m}}$ of the aggregates, as predicted by Eq. (22).

\section{CONCLUSIONS}

In this paper we have reviewed the principles of the HNFS technique and proposed a method of data analysis that significantly improves its sensitivity and accuracy. The method greatly relaxes the requirements on the optical/ mechanical stability of the instrumental setup, allowing the technique to be profitably used for both particle sizing appli- cations and characterization of nonstationary systems undergoing irreversible growth processes. When compared with traditional low-angle light scattering methods, this technique appears to be much more sensitive and accurate, free of all the nasty problems associated with the stray-light subtraction always present in traditional LALS.

The technique was tested by using calibrated colloidal solutions, made of either stable or aggregating colloids. By using an iterative inversion algorithm based on the Mie theory, we have been able to recover with high accuracy the size distributions of both monodisperse and bimodal samples with particle diameters in the range $1-10 \mu \mathrm{m}$. The flocculation kinetics of small colloids undergoing a reaction limited aggregation process was also successfully studied, obtaining results quite consistent with the prediction expected for a RLA process.

Finally we pointed out that the HNFS technique, in conjunction with the new method of data analysis devised in this work, is a potential method for carrying out also low-angle dynamic light scattering. Thus simultaneous static and dynamic light scattering measurements would become feasible, outstandingly increasing the potential of the technique.

\section{ACKNOWLEDGMENTS}

We thank D. Brogioli for helpful discussions. This work was supported by funds from Istituto Nazionale per le Fisica della Materia (INFM), Project PAIS-NFS.
[1] M. Giglio, M. Carpineti, and A. Vailati, Phys. Rev. Lett. 85, 1416 (2000).

[2] M. Giglio, M. Carpineti, A. Vailati, and D. Brogioli, Appl. Opt. 40, 4036 (2001).

[3] D. Brogioli, A. Vailati, and M. Giglio, Appl. Phys. Lett. 81, 4109 (2002).

[4] D. Brogioli, A. Vailati, and M. Giglio, Europhys. Lett. 63, 220 (2003).

[5] M. Carpineti, F. Ferri, M. Giglio, E. Paganini, and U. Perini, Phys. Rev. A 42, 7347 (1990).

[6] F. Ferri, Rev. Sci. Instrum. 68, 2265 (1997).

[7] J. W. Goodman, Introduction to Fourier Optics (McGraw-Hill, New York, 1996).
[8] J. R. de Bruyn, E. Bodenschatz, S. W. Morris, S. P. Trainoff, Y. Hu, D. S. Cannell, and G. Ahlers. Rev. Sci. Instrum. 67, 2043 (1996).

[9] M. Giglio, D. Brogioli, M. Potenza, and A. Vailati, Phys. Chem. Chem. Phys. 7, 1547 (2004).

[10] F. Ferri, A. Bassini, and E. Paganini, Appl. Opt. 34, 5829 (1995).

[11] F. Ferri, G. Righini, and E. Paganini, Appl. Opt. 36, 7539 (1997)

[12] D. Asnaghi, M. Carpineti, M. Giglio, and M. Sozzi, Phys. Rev. A 45, 1018 (1992).

[13] M. E. Fisher and R. J. Burford, Phys. Rev. 156, 583 (1967). 